\title{
Cloning and Characterization of the Gene Encoding 3- hydroxy-3- Methylglutaryl-coenzyme A (HMG-CoA) Reductase from Fritillaria Cirrhosa D. Don
}

\author{
Qi Zhao' ${ }^{*}$, Rui Li', Xiao Chen', Qian Yang', Jian Li ${ }^{1}$ \\ ${ }^{I}$ Chengdu University Chengdu, Sichuan, China
}

\begin{abstract}
The enzyme 3-hydroxy-3-methylglutaryl-CoA reductase (HMGR; EC1.1.1.34) catalyzes the first committed step of isoprenoids biosynthesis in Mevalonate (MVA) pathway. Here we report for the first time the cloning and characterization of a full-length cDNA encoding HMGR from Fritillaria cirrhosa (FcHMGR), a bulbous medicinal plant. The full-length cDNA of FcHMGR was 2072 base pair (bp), containing a 1680-bp open reading frame. Bioinformatical analyses revealed that FCHMGR had HMG CoA-binding domains and two NADPH binding domains, which are required for HMGR activity. Quantitative real-time PCR (qRT-PCR) analysis revealed that FcHMGR expressed high in mature bulbs. A truncated version of FcHMGR protein lacking the N-terminal 249-bp $G C$ rich area was expressed in Escherichia coli. The crude cell lysate containing the recombinant protein showed a better HMGR activity than the control and the relative enzyme activity was calculated to be $1.62 \mathrm{U} / \mathrm{mg}$. The cloning, characterization and functional analysis of FcHMGR gene allowed us to further understand the role of FcHMGR involved in steroidal alkaloid biosynthetic pathway in F. cirrhosa at the molecular level.
\end{abstract}

Keywords: 3-Hydroxy-3-methylglutaryl-CoA reductases, Fritillaria cirrhosa, Molecular cloning, Expression pattern, Prokaryotic expression

*Author for correspondence: zhaoqi@cdu.edu.cn 


\section{INTRODUCTION}

Fritillaria cirrhosa D. Don (Family, Liliaceae) is a bulbous medicinal and perennial herbaceou plant, which is native to the southeastern margin of the Qinghai-Tibet Plateau region of China1. It has been widely used in traditional Chinese medicine (TCM) for over 2,000 years as a curative therapy for chronic cough. Steroidal alkaloids are the main active secondary metabolites in F. cirrhosa. So far, at least twenty kinds of monomeric alkaloids, including peimine, imperialine, verticine, verticinone, and peimisine, have been isolated from this plant2. Previous studies have demonstrated a wide array of pharmacological activities of these alkaloids, including anti-inflammatory and anti-cancer3. The content of alkaloids in F. cirrhosa were reported to be very low, limiting its medical application. Biotechnological strategies have been exploited to increase the yield of alkaloids from the cultured bulbs4 or via cell suspension cultures5. Overexpression of steroidal alkaloids biosynthesis-related genes combining with bulbs culturing is considered to be a practical way to stimulate the secondary metabolites production of $\mathrm{F}$. cirrhosa.

The first key regulatory, rate-limiting enzyme in the mevalonate (MVA) pathway for the biosynthesis of phytosterol in plants is 3-hydroxy-3-methylglutaryl-coenzyme A reductase (HMGR), which catalyzes the conversion of HMG-CoA to MVA7. The MVA pathway is the main route for the production of steroidal alkaloid backbones8. Because of its critical role in regulating sterol biosynthesis, HMGR has been studied as a rational candidate for phytosterol accumulation of plants. In Nicotiana tabacum L., the heterologous expression of Withania somnifera HMGR has been showed to be positively correlated with the accumulation of phytosteroids. Transgenic tobacco expressing an N-terminal truncated Hevea brasiliensis HMGR (t-HMGR) had up to 10 -fold leaf phytosterol sterol levels. Moreover, it has been reported that characterization of a sterol-overproducing tobacco mutant showed a 3-fold increase in HMGR activity in leaf tissue comparing to the wild-type tissue9. These results indicate that the expression of HMGR gene is linked to the accumulation of phytosteroid in plants.

In this study, we cloned FcHMGR cDNA from F. cirrhosa bulbs, performed a bioinformatics analysis and studied its expression patterns. The functionality of the FcHMGR gene was determined in prokaryotic (Escherichia coli) expression model systems.

\section{MATERIAL AND METHODS}

\section{Plant materials}

The F. cirrhosa mature bulbs, roots, stems and leaves were collected from 3-year-old plants grown in the origin of the Kangding fold-thrust belt mountains (located at 30³'44.9" N, 101 $58^{\prime} 3.81$ " E, altitude $4300 \mathrm{~m}$ ) in Sichuan Province, China, then snap frozen in liquid nitrogen and transferred to a $-80{ }^{\circ} \mathrm{C}$ freezer till further use for comparative tissue specific expression profile.

\section{Cloning and sequence analysis of the FcHMGR cDNA}

Total RNA was extracted from the bulbs of $F$. cirrhosa using Trizol reagent (Invitrogen, Carlsbad, CA, USA). Reverse transcription of pooled RNA was carried out with oligodeoxythymidine using the PrimeScript ${ }^{\mathrm{TM}}$ RT Reagent Kit (TaKaRa, Dalian, China) according to the manufacturer's instructions. The degenerate primers, HMGRF and HMGRR (Table1), were designed based on the conserved motifs of the 
plant HMGR proteins. For 3' and 5' RACE, three universal primers [oligo(dT)18, AP1, and AP2] and three gene-specific primers (HMGR3', HMGR5'RT and HMGR5', Table 1) were used in the rapid amplification of cDNA ends (RACE)10. Based on the alignment of the 3 ' and 5' RACE fragments, primers (HMGR-ORFF and HMGRORFR) (Table 1) were designed to obtain the cDNA of FcHMGR gene. The products were cloned into pEASY-T vectors (TransGene) and sequenced. Sequences were examined for similarities with sequences in the GenBank database using the BLAST program (http://blast.ncbi.nlm.nih.gov/Blast.cgi). The amino acid sequences of cloned cDNA fragments were deduced and protein sequences were aligned using the program DNAMAN 6.0 (http://www.lynnon.com). The protein physical properties analysis were using the On-line Analysis System (http://web.expasy.org/compute_pi/). Phylogenetic relationship was analyzed by multiple alignments of HMGR proteins using the MEGA 4.1 program11. The protein tertiary structure was predicted by Swiss-model tool (http://swissmodel.expasy.org/interactive).

Table. 1. Primers used for PCR in this study

\begin{tabular}{ccc}
\hline Primers & Sequences (5'-3') & Usage \\
\hline HMGRF & THYTNGGNCARTGYTGYGARATGCC & Cloning of $F c H M G R$ cDNA \\
HMGRR & CDATCCARTTNACNGCNGGYTT & Cloning of $F c H M G R$ cDNA \\
AP1 & GTCAACGATACGCTACGTAACG & Anchor \\
AP2 & TACGTAACGGCATGACAGTG & Anchor \\
HMGR3' & TCTGTGACCATGCCATCTA & 3' RACE \\
HMGR5'RT & GTACTCGCAACCAAACACCCCT & 5' RACE \\
HMGR5' & TTCGATTCGAGTATATATG & 5' RACE \\
HMGR-ORFF & ATGGACATCCGCCGCCG & Cloning of $F c H M G R$ ORF \\
HMGR-ORFR & TCAAGAGCCAGCCTTAGACATATCT & Cloning of $F c H M G R$ ORF \\
HMGRqF & GCTCCAAGGTATTCAATGCTCA & Quantitative Real-time PCR \\
HMGRqR & CGATGACTGCCTCACATACCA & Quantitative Real-time PCR \\
18SqF & TACGACTCTCGGCAACGGA & Quantitative Real-time PCR \\
18SqR & CAAAGGGGCAATGGGAACA & Quantitative Real-time PCR \\
Nt-HMGR-GEXF & CGCGGATCCATGATCTACCTCATCAGCTTCTTC & Construction of the pGEX vector \\
\hline Nt-HMR-GEXR & CGGATTCTCAAGAGCCAGCCTTAGACATATCT & Construction of the pGEX vector \\
\hline
\end{tabular}

\section{Expression analysis of FcHMGR by real-time quantitative PCR}

Total RNA was extracted using the same method as described above. Approximately $0.5 \mu \mathrm{g}$ of the total RNA from each pool from bulbs, roots, stems and leaves were reverse transcribed into cDNA with oligodeoxythymidine as primer in a volume of 10 $\mu \mathrm{L}$ following the PrimeScriptTM RT Reagent Kit protocol. The mixture was diluted to $100 \mu \mathrm{L}$ with sterile water and the synthesized DNA was used as template in quantitative real-time PCR (qRT-PCR). Gene-specific primers (HMGRqF and HMGRqR (Table 1) were designed to amplify the specific cDNA fragment of FcHMGR and the expected size of the RT-PCR product is $248 \mathrm{bp}$. The $18 \mathrm{~S}$ gene (GeneBank accession no. AY616727.1) of Fritillaria cirrhosa was used as the internal 
reference. All qRT-PCRs were performed using SYBR ${ }^{\circledR}$ Premix Ex Taq ${ }^{\mathrm{TM}}$ II (TaKaRa) and a Bio-Rad CFX96 Real-time PCR machine. The PCR protocol was 95 ${ }^{\circ} \mathrm{C}$ for $10 \mathrm{~s}$ initially, followed by 40 cycles, each comprising $95{ }^{\circ} \mathrm{C}$ for $10 \mathrm{~s}, 58{ }^{\circ} \mathrm{C}$ for $20 \mathrm{~s}$ and $72{ }^{\circ} \mathrm{C}$ for $20 \mathrm{~s}$, then $3 \mathrm{~min}$ at $72{ }^{\circ} \mathrm{C}$.. At least three independent replicates of each experiment were performed.

\section{Expression of N-terminal truncated FcHMGR (FcNt-HMGR) in E.coli BL21}

Primers Nt-HMGR-GEXF and Nt-HMGR-GEXR (Table 1) were designed and synthesized to amplify the coding sequence of N-terminal truncated FcHMGR which lacking a 249-bp GC enrichment area. The resulting fragment was digested with BamH I/EcoR I and ligated into the corresponding restriction sites of the expression vector pGEX6p-1. The recombinant plasmid pGEX-FcNt-HMGR was transformed into E.coli strain BL21 (DE3) for protein expression.

Three colonies of E.coli strain BL21 cells harboring the recombinant plasmid pGEXFcNt-HMGR were inoculated at $37^{\circ} \mathrm{C}$ in LB medium containing ampicillin (100 mg l$1)$, with shaking $(220 \mathrm{rpm})$ until the cell cultures at OD600 value of 0.6. Then the protein expression was induced by addition of isopropyl- $\beta$-D-thiogalactoside (IPTG) to a final concentration of $0.25 \mathrm{mM}$ and the cultures were grown for an additional $4 \mathrm{~h}$. Over-expressed proteins were detected by analyzing total protein on $12 \%$ sodium dodecylsulfate polyacrylamide gel electrophoresis (SDS-PAGE) followed by coomassie brilliant blue R250 staining.

\section{Determination of the FcNt-HMGR relative enzyme activity}

Cells containing FcNt-HMGR were induced at $37^{\circ} \mathrm{C}$ for $4 \mathrm{~h}$ and harvested by centrifugation at $5,000 \mathrm{~g}$ for $15 \mathrm{~min}$ and immediately frozen at $-80^{\circ} \mathrm{C}$. Frozen cell pellets were thawed and suspended in protein binding buffer $(30 \mathrm{mM}$ Tris- $\mathrm{HCl}, \mathrm{pH} 7.5$, $5 \%$ glycerol, $100 \mathrm{mM} \mathrm{NaCl}, 1 \mathrm{mM}$ PMSF). The cells were then lysed by sonication on ice. Cellular debris was removed by centrifugation at $4^{\circ} \mathrm{C}, 20,000 \mathrm{~g}$ for $30 \mathrm{~min}$. Supernatant containing FcNt-HMGR was subjected to enzyme activity assay according to the method of $\mathrm{Li}$ et al with minor modifications 12. Enzymatic activities were assayed in $200 \mu \mathrm{l}$ of reaction mixture consisting of $50 \mathrm{mM}$ Tris- $\mathrm{HCl}$ buffer $(\mathrm{pH}$ 7.0), $0.075 \mathrm{mM} \mathrm{NAD+}, 4 \mathrm{mM}$ DTT, and crude cell lysate. The substrate 3-Hydroxy-3methylglutaryl coenzyme A (Sigma) was added to the reaction mixtures after preincubation for $10 \mathrm{~min}$ at $37^{\circ} \mathrm{C}$ to start the reactions. Enzyme activities were determined according to the absorbance at $340 \mathrm{~nm}$ (reflecting the consumption of $\mathrm{NAD}+$ ). One unit of the activity was defined as $1 \mu \mathrm{mol} / \mathrm{min}$ of NAD+ consumption. Each determination was carried out at least three times. The supernatant of the culture without IPTG induction was used as a negative control. Protein concentration was measured by bicinchoninic acid (BCA) method 13 using a protein assay kit (Thermo, USA). All assays were repeated at least three times.

\section{RESULTS}

\section{Cloning and sequence analysis of FcHMGR}

The full-length cDNA of the FcHMGR gene had a size of 2072 bp with 5' and 3' untranslated regions (UTRs) and a polyA tail (Figure 1). The open reading frame (ORF) was flanked by a 60-bp 5-UTR and a 332-bp 3-UTR. An ORF search showed that FcHMGR contained a 1680 bp ORF encoding a protein with 559 amino acids (Figure 1, 2). The calculated molecular weight of the encoded protein was 
approximately $59.2 \mathrm{kDa}$, with a theoretical isoelectric point of 7.48. Using the amino acid sequence as query, the sequence similarities database search (NCBI) showed high homology to several HMGR-like proteins including the Phoenix dactylifera HMGR (80\% identity), Elaeis guineensis HMGR(80\% identity), Brachypodium distachyon HMGR $(80 \%$ identity), Sorghum bicolor HMGR(78\% identity) and Dendrobium huoshanense HMGR(76\% identity). DNAMAN 6.0 software was used to carry on the homologous comparison and the results are shown in Figure3, we can see from the figure that the sequence of amino acids of the higher plant HMGR reaches low homology from 150 amino acids downstream of the N-terminal, followed by a highly conserved region, which contains two HMG CoA - binding domains (EMP (V/I) GY (V/I) QIP, TTEGCLVA) and two NADPH binding domain (DAMGMNM). A comparative 3D modeling of FcHMGR catalytic domain was performed using SwissModel with the HMGR (PDB no. 1dqa.1.A; sequence identity is 58.39\%) with Homo sapiens HMGR protein as the template. The space layout of HMGR function area is folded into a "V" shape. The $\mathrm{L}$ structure domain in the middle possesses two functions: HMG-CoA binding motif and NADPH biding motif (Figure3, 4B). Phylogenetic analysis showed that the evolutionary tree clustering has two kinds, monocots and dicotyledonous. FcHMGR was closely related to the Dendrobium huoshanense HMGR, which is in the same sort of angiosperm and monocotyledon, clusters (Figure4A).

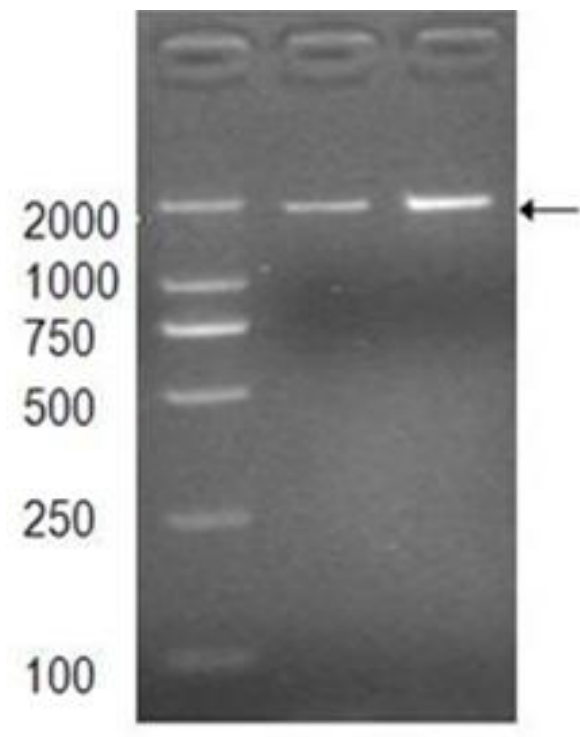

A

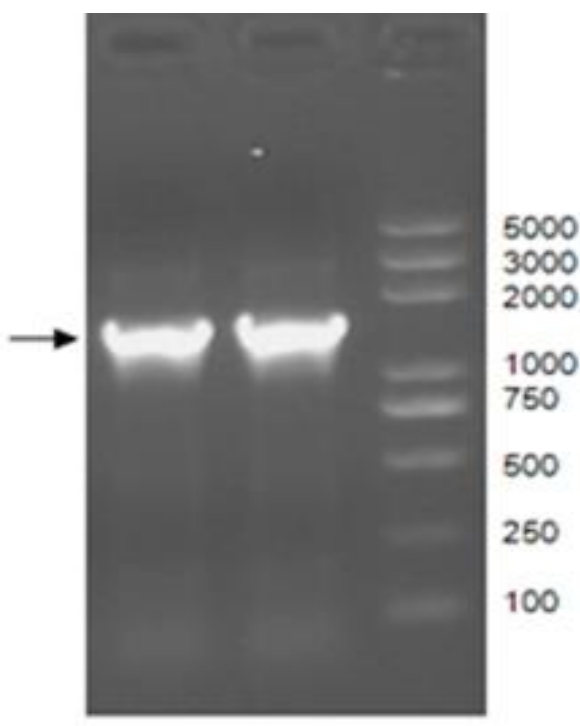

B

Figure 1 Amplification of $F c H M G R$ full-length cDNA (A) and ORF (B). 
GATTCCACACACCTTTCGCGGCGCGGAGGAAGGCGCGGCGCGGTTCCTCCTCCCCTCCCG 60 ATGGACATCCGCCGCCGCAAACCCTCCACCCGCCCGCCTCCGGCGGCGGCGCCGCCCCTC 120 $\begin{array}{llllllllllllllllllll}M & D & I & R & R & R & K & P & S & T & R & P & P & P & A & A & A & P & P & L\end{array}$ CCCTCCCGCATCCAAGCCTCTGACGCTCTCCCTCTCCCCATCCGCCACACCAACCTTCTC 180

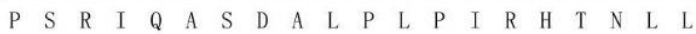
TTCTCCGCCCTCTTCGCCGCCTCCCTCGTCTTCCTCATGCGCCGGTGGCGCGAAAAGGTC 240 $\begin{array}{llllllllllllllllllll}\text { F } & S & A & \text { L } & \text { F } & \text { A } & \text { A } & \text { S } & \text { L } & V & \text { F } & \text { L } & \text { M } & R & R & \text { W } & R & \text { E } & K & V\end{array}$ CGCGTCTCCGCCCCCCTCCACGTCATCGGCCTCGCCGACATGGCCGCCATGATCTGCCTC 300 \begin{tabular}{llllllllllllllllllll}
\hline$R$ & $V$ & $S$ & $A$ & $P$ & $L$ & $H$ & $V$ & $I$ & $G$ & L & A & D & M & A & A & M & I & C & L
\end{tabular} GTCGCTTCCCTCATCTACCTCATCAGCTTCTTCGGGATCGCCTACGTCCAGTCCGTCGTC 360 $\begin{array}{llllllllllllllllllll}V & \text { A } & \text { S } & \text { L } & \text { I } & Y & \text { L } & \text { I } & S & \text { F } & \text { F } & G & \text { I } & \text { A } & \text { Y } & V & \text { Q } & \text { S } & \text { V } & \text { V }\end{array}$ TCCTCACACGAGGATGACGACGACTTCCTCCTCCCCTCGCCGGCGCAGCCGGCCCCGCCG 420

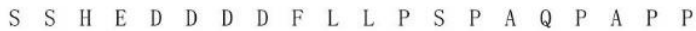
GCCGCGCCGACTCAATGCCCCCTCCTCCGCTCGGACAAAGAATCAATCTCAGTACCGGCT 480 $\begin{array}{llllllllllllllllllll}\text { A } & \text { A } & P & \text { T } & Q & C & P & L & L & R & S & D & K & E & S & I & S & V & P & A\end{array}$ GTCGATGCGAGTGATGAAGACATAATCGCCTCAGTGGTGTCCGGTAAAACACCTTCATAT 540 $\begin{array}{llllllllllllllllllll}V & D & A & S & D & E & D & I & I & A & S & V & V & S & G & K & T & P & S & Y\end{array}$ ATACTCGAATCGAAGCTGGGCGACTGCAGGCGGGCCGTTGCGATCCGGCGTGAGGCGCTG 600

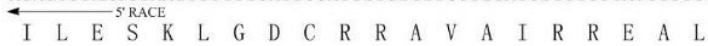
CGGCGGATGACCGGCCGGCCGATGGAGGGGCTGCCAATTGAGGGGTTTGACTATGCATCG 660 $\begin{array}{llllllllllllllllllll}R & R & M & T & G & R & P & M & E & G & L & P & I & E & G & F & D & Y & A & S\end{array}$ ATCCTGGGGCAGTGCTGTGAGTTGCCGATTGGGTATGTTCAGCTCCCGGTGGGGATTGCT 720

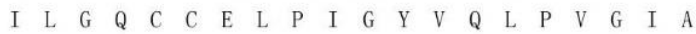
GGGCCACTGGCCCTGGATGGGAGGGAGTTCTATGTGCCGATGGCGACCACTGAGGGGTGT 780

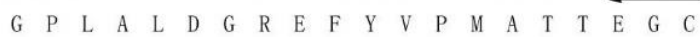
TTGGTTGCGAGTACAAACAGGGGTTGTAAAGCTATATTGGAGTCGGGCGGGGCGATGAGT 840

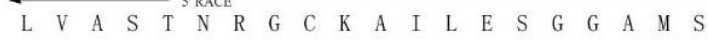
GTGGTGTTGAAGGATGGGATGACGCGGGCGCCGGCGGTGAGGTTTTCGACGGCGATGAGG 900

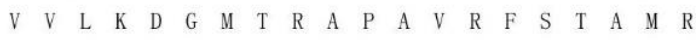
GCGGCTGAGCTCAAGTTCTTCTTGGAGGAGCCAGAGAACTTTGATACACTGGCTGTTGTC 960 $\begin{array}{llllllllllllllllllll}\text { A } & \text { A } & \text { E } & \text { L } & \text { K } & \text { F } & \text { F } & \text { L } & \text { E } & \text { E } & \text { P } & \text { E } & \text { N } & \text { F } & \text { D } & \text { T } & \text { L } & \text { A } & V & \text { V }\end{array}$ TTCAATAGGTCGAGCAGATTTGCAAGGCTCCAAGGTATTCAATGCTCACTCGCAGGGAGG 1020

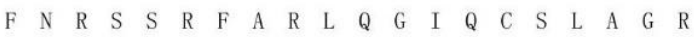

TTCAATAGGTCGAGCAGATTTGCAAGGCTCCAAGGTATTCAATGCTCACTCGCAGGGAGG 1020 $\begin{array}{lllllllllllllllllllll}\text { F } & N & R & S & S & R & F & A & R & L & Q & G & I & Q & C & S & L & A & G & R\end{array}$ AATCTCTACATGAGATTCTGCTGTAGCACAGGTGATGCCATGGGGATGAATATGGTGTCC 1080

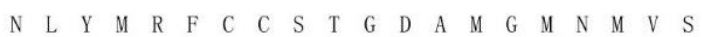
AAGGGAGTGCAGAATGTGTTGGATTATCTTCAGAACGATTTTCCGGATATGGATGTGGTC 1140 $\begin{array}{lllllllllllllllllllll}K & G & V & Q & N & V & \text { L } & D & Y & L & Q & N & D & F & P & D & M & D & V & V\end{array}$ AGCATCTCAGGGAATTTCTGTTCGGACAAGAAGCCTGCTGCTGTAAACTGGATCGAAGGG 1200

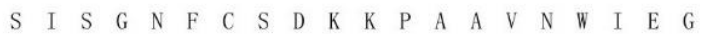
AGAGGCAAATCAGTGGTATGTGAGGCAGTCATCGGGGAAGATGTGGTGAAGAAGGTCCTG 1260 $\begin{array}{llllllllllllllllllll}R & G & K & S & V & V & C & E & A & V & I & G & E & D & V & V & K & K & V & L\end{array}$ AAGACCACCGTGCCTGCTCTGGTAGAGCTGAACATGATCAAGAATCTCGCTGGCTCAGCT 1320 $\begin{array}{llllllllllllllllllll}\text { K } & \text { T } & \text { T } & \text { V } & \text { P } & \text { A } & \text { L } & \text { V } & \text { E } & \text { L } & \text { N } & \text { M } & \text { I } & \text { K } & \text { N } & \text { L } & \text { A } & \text { G } & \text { S } & \text { A }\end{array}$ GTCGCCGGTGCCTTGGGGGGATTCAACGCCCACGCCAGCAACATCGTGTCTGCCGTCTTC 1380

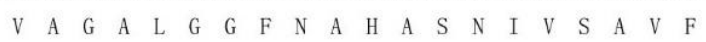
ATTGCCACTGGCCAAGATCCCGCCCAGAACGTCGAGAGCTCTCACTGCATCACCATGATG 1440

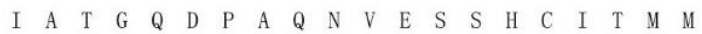
GAGGCTGTGAATGGCGGGAAAGATCTTCATGTCTCTGTGACCATGCCATCTATTGAGGTT 1500

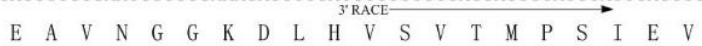
GGTACAGTTGGGGGTGGGACCCAGCTGGCGGCTCAGGCTGCTTGCTTGGATCTCCTCGGT 1560

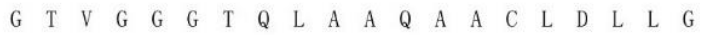
GTGAAGGGCGCAAGCATGGAGTCACCTGGAGCCAATGCGAGGCTCCTGGCTACTATCATA 1620 $\begin{array}{llllllllllllllllllll}V & K & G & A & S & M & E & S & P & G & A & N & A & R & L & L & A & T & I & \text { I }\end{array}$ GCTGGCGCCGTTTTAGCCGGGGAGCTCTCTCTCATGTCGGCCCTTGCGGCTGGTCAGCTC 1680

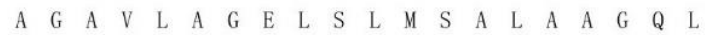
GTGAAGAGCCACATGAAGTATAACAGGTCCAGCAAAGATATGTCTAAGGCTGGCTCTTGA 1740

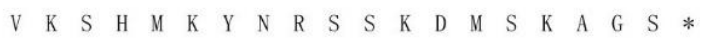
GATGCTCCCTCGTCGTCGACGGTGATAATTTAATTTGTTTGTTGTTTGTTAAGGCGAATT 1800 CGAGGAAGTAGTAATAATGGACGATGTAAGTCTGATGTTGAGTGTCTCCCTAGATGTGTT 1860 TCTTATGCTTAACCATGTGATCAACAGACTGTCATGTTTGTTTTACTTTTCATAGGACAT 1920 GAGAAGCACATGTAGGGAATCCTCTGGATCTGGTTTCTGAGCTGCTTGTATTTTGTATCT 1980 CTATGTCAGTCTCAAATGTATTATTTCTTCTGTGTCTTGAGCAATGAATAAAATACTTAT 2040 CTATGTGTGTCTGTCGGTTTGTCCTAAAAAAA 2072

Figure 2 Nucleotide and the deduced amino acid sequence of $F c H M G R$ from $F$. cirrhosa.

(The N-terminal truncated region is underlined; The 3' and 5' RACE primers were marked with forward and reverse solid arrows, respectively. Asterisk denotes translation terminator) 

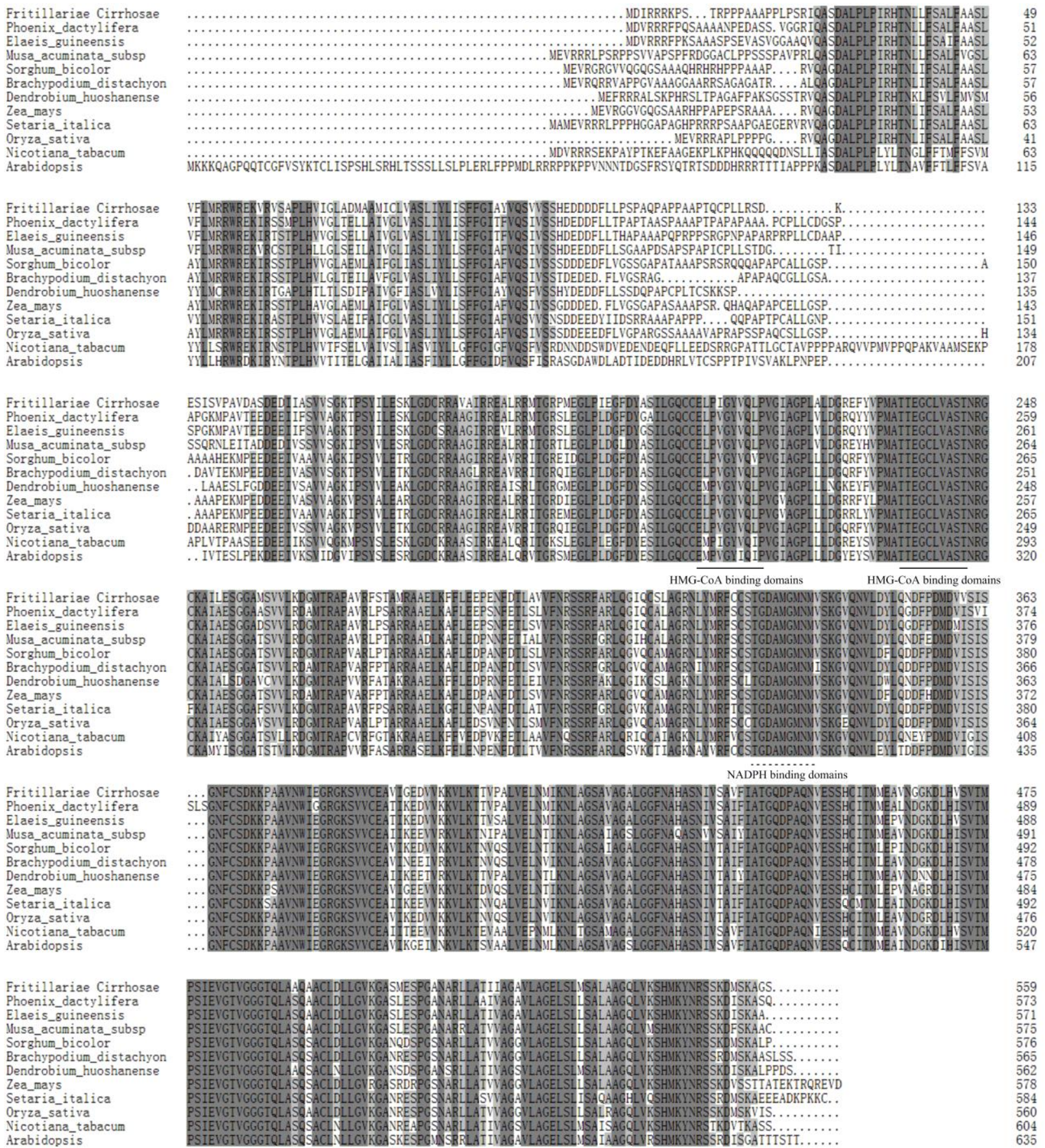

559
573
571
575
576
565
562
578
584
560
604
635

Figure 3 Multiple alignment of FcHMGR proteins from several species.

(The HMG-CoA binding domains and NADPH binding domains were marked with solid line and dotted line, respectively. The highly conserved regions of the alignments have been shaded) 


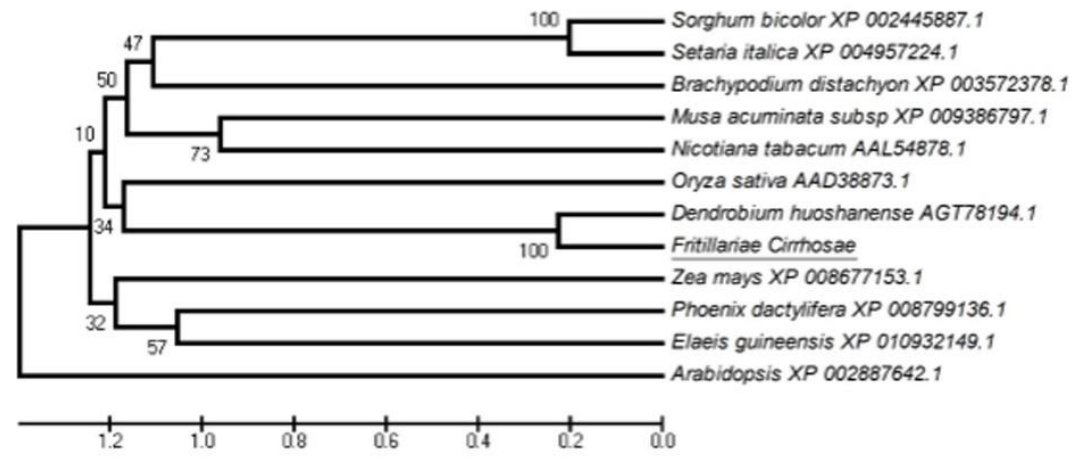

A

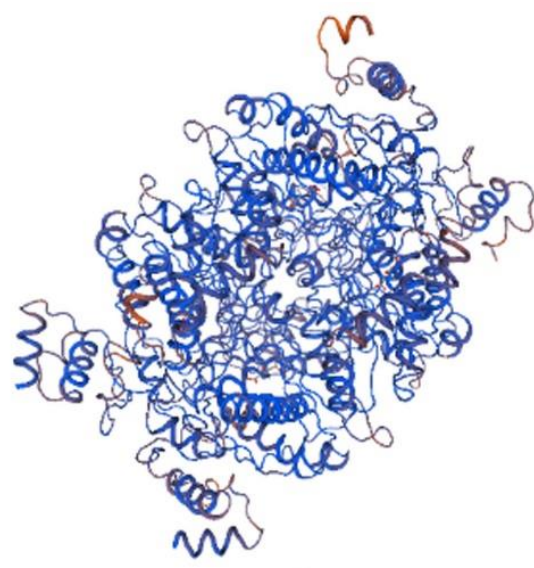

B

Figure 4 FcHMGR protein phylogenetic and tertiary structure analysis.

(A) Phylogenetic tree of FcHMGR protein sequences from different species.

(B) FcHMGR protein tertiary structure.

\section{Transcription profile of $\mathrm{FcHMGR}$}

To better understand the expression of FcHMGR, we further analyzed the expression patterns of FcHMGR in bulbs, roots, stems and leaves of 3-year-old F. cirrhosa seedlings. The transcription level of FcHMGR was determined by qRT-PCR. Results indicated that FcHMGR were expressed in all examined tissues, with the highest expression in the bulb. The expression level of FcHMGR in leaf were 0.6 fold lower than that in bulb (Figure 5). This result indicated that expression of FcHMGR in vegetative tissues was non-tissue-specific.

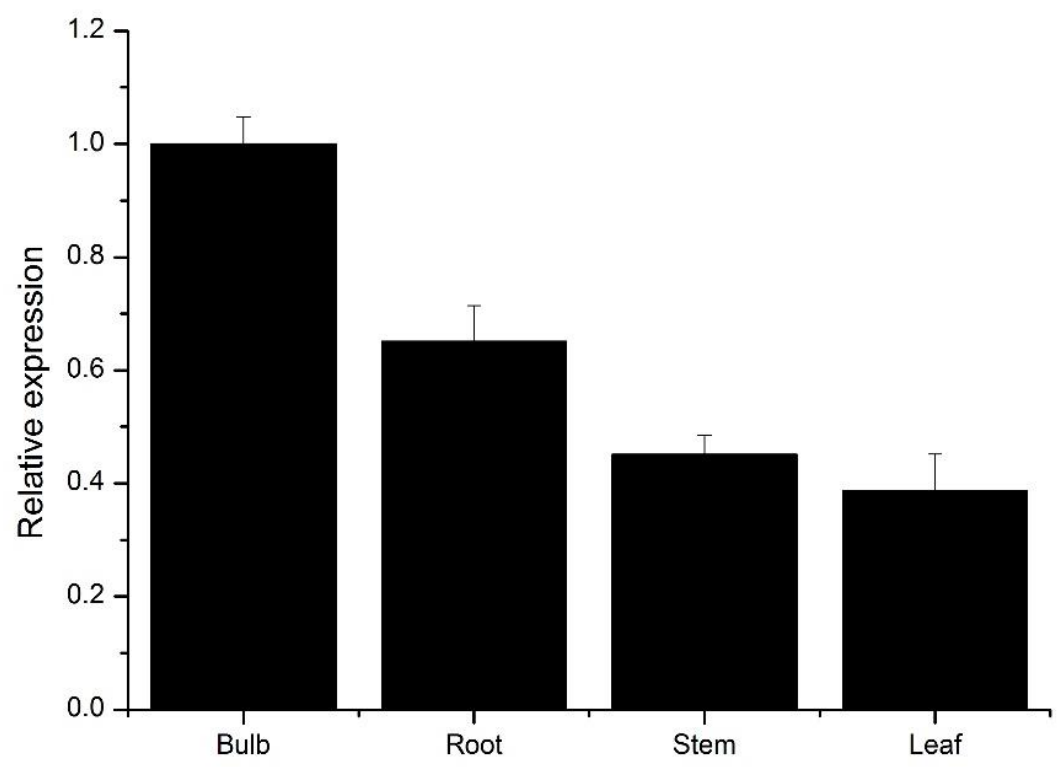

Figure 5 Spatial expression patterns of $F c H M G R$ gene measured by qRT-PCR. 


\section{Expression and enzyme activity assay of the recombinant FcNt-HMGR in E.coli BL21}

We previously found that the expression of the full length recombinant FcHMGR in E.coli was not detectable (data not shown), presumably due to the GC-rich region near the N-terminal of the sequence. We sub-cloned an N-terminal 249-bp truncated FcHMGR (FcNt-HMGR) into pGEX-6p-1 for recombinant protein expression. The Nterminal 83 amino acids of FcHMGR showed low homology with plant HMGR families and had no predicted enzyme activity domains. Subsequent prokaryotic expression assays proved that this recombinant FcNt-HMGR can be successfully expressed as soluble protein in E.coli BL21 strain under IPTG iudction, which was not present in the extracts obtained from non-induced E. coli cells (Figure 6A). Because of the GST-tag fusion, molecular masses of the FcNt-HMGR proteins was $75 \mathrm{kDa}$.

To determine whether the recombinant FcNt-HMGR protein was a functional HMGR protein, the enzyme activity of FcNt-HMGR was measured according to the absorbance attenuation of NADPH at $340 \mathrm{~nm}$. As shown in Figure 6B, when the supernatant containing FcNt-HMGR was added into the reaction solution, the NADPH is rapidly consumed, resulting in a sharp decline of absorbance at $340 \mathrm{nM}$. The absorbance of the control (non-induced FcNt-HMGR E. coli cells) showed no dramatic change. This indicated that the heterologous expressed FcNt-HMGR protein had a better reductase activity than the control and the relative enzyme activity was calculated to be $1.62 \mathrm{U} / \mathrm{mg}$.

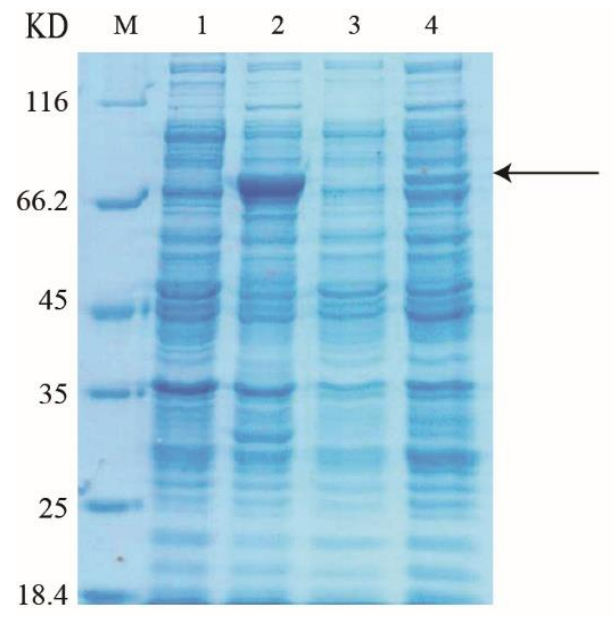

A

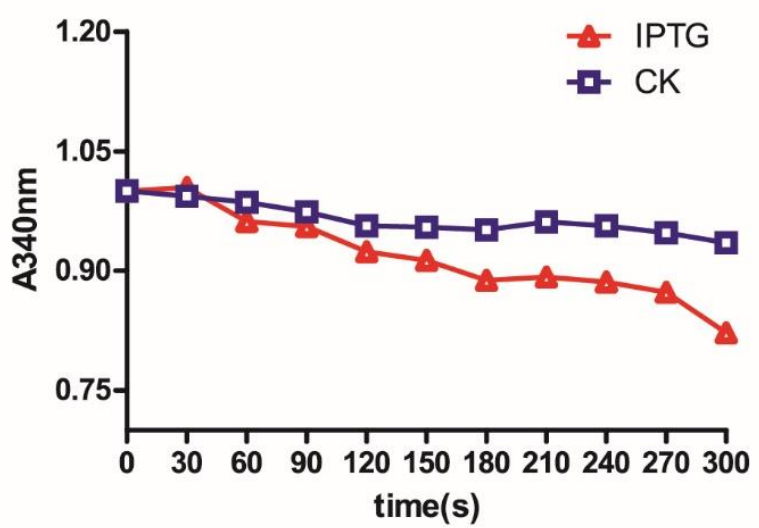

B

Figure 6 Recombinant FcNT-HMGR protein expression in BL21DE3 cells (A) and enzyme activity assay (B)

(A) SDS-PAGE (12\%) analysis of FcNt-HMGR over-expression in E.coli BL21. Lane M, protein marker (Beyotime p0061); lane 1, without IPTG induction; lane 2, IPTG induction (arrow mark is $75 \mathrm{kDa}$ ); lane 3, the supernatant of lysate without IPTG induction; lane 4, the supernatant of lysate with IPTG induction. (B) The enzyme activity assay by measuring the absorbance attenuation of NADPH at $340 \mathrm{~nm}$. CK, non-induced recombinant FcNt-HMGR BL21 cells; IPTG, induced recombinant FcNt-HMGR BL21 cells.

\section{DISCUSSION}

HMGR are generally conserved in land plants, playing an important role in the regulation of secondary metabolism of the phytosteroid7,14. In this study, we isolated a gene, FcHMGR, encoding a HMGR-like protein from F. cirrhosa. Homologous and phylogenetic analysis indicated that the FcHMGR protein has the typical polypeptide sites which are required for HMGR activity, including two HMG CoA - binding 
domains (EMP (V/I) GY (V/I) QIP, TTEGCLVA) and two NADPH binding domains (DAMGMNM). Eti et al. found that the glutamic acid of TTEGCLVA plays a crucial role in the HMGR catalytic process 15 . Homology modeling demonstrated that the main body of the space layout of HMGR function area was folded into a "V" shape. The structure domains of L in the middle part possess the HMG-CoA binding motif and NADPH binding motif16. Combining with the tertiary structure prediction analysis (Figure3,4B), these results sugessted that FcHMGR is a functional protein that plays a role in the biosynthetic pathway of phytosteroid.

Transcription analysis is a useful tool for deciphering gene functions. The bulbs of F. cirrhosa are used for medicinal purposes17. qRT-PCR showed that the basal FcHMGR mRNA levels was high in mature bulbs (Figure 5), consistent with its high level of phytochemicals18, suggesting a positive correlation between the expression of this FcHMGR-like genes and accumulation of phytochemicals in F. cirrhosa bulbs.

We exploited the prokaryotic expression system to further evaluate the enzymatic activity of the protein encoded by this gene. Initially, the full protein of FcHMGR could not be expressed in E.coli, which was most likely related the GC enrichment region at the N-terminal of the gene. So, we deleted 249-bp from this region of FcHMGR to verify whether this change will affect the recombinant protein expression and function. Subsequent experiments showed this $\mathrm{N}$-terminal truncation was in favor of Fc-HMGR expression. The recombinant protein showed reductase activity according to the result from out in vitro assay (Figure 6). This result also suggested that the function of Fc-HMGR depends on its conserved structure. The low homology region, including the N-terminal 249-bp truncated area in this study, caused no change of the protein function.

\section{CONCLUSION}

In conclusion, based on the results presented in this study, the correlation between phytochemicals content and FcHMGR gene expression level in F. cirrhosa bulbs have been established to some extent, which needs to be further verified in studies with larger samples. Some functional information was also obtained according to the deduced amino acid sequence of the FcHMGR enzyme. We also evaluated the functional validity of FcHMGR in the in vitro enzymatic reaction, but few correspond to its molecular mechanisms responsible for substrate specificity still need to be clarified, CRISPR/Cas9 mutagenesis technology will be a useful method for such a study 19, which may be conducted in the future. On the basis of this study, the HMGR enzyme of F. cirrhosa could be a good candidate for the engineering of the F. cirrhosa phytochemicals synthesis pathway.

\section{ACKNOWLEDGMENTS}

This work was supported by the Natural Science Foundation of China (31600261) and the Sichuan Provincial Department of Education Natural Science Key Project (17ZA0078).

\section{REFERENCES}

1. Zhang DQ, Gao LM, Yang YP. Genetic diversity and structure of a traditional Chinese medicinal plant species, Fritillaria cirrhosa (Liliaceae) in southwest China and implications for its conservation. Biochem Syst Ecol. 2010; 38(2):236-242.

2. Li HJ, Jiang Y, Li P. Chemistry, bioactivity and geographical diversity of steroidal alkaloids from the Liliaceae family. Cheminform. 2007; 38(5):735-752. 
3. Luo H, Zhang L, Xu W, Yang J, Yang W, Yang A, et al. Simultaneous Determination of Four Main Isosteroidal Alkaloids of Bulbus Fritillariae Cirrhosae in Rat Plasma by LC-MS-MS. Chromatographia. 2012; 75(13-14):729-737.

4. Yang Y, Jiang H, Hua-Long FU, Lan LQ. Determination and histochemical localization of alkaloids content in wild and tissue cultured Fritillaria cirrhosa D. Don. Journal of Sichuan University. 2008; 45(1):209-213.

5. Wang YH, He ZS, Sun YX, Ma LL, Liu YL, Lin KX. Study on the production of alkaloid by cell mass suspension culture of Fritillaria cirrhosa. Journal of Chinese Medicinal Materials. 2011; 34(2):183-186.

6. Sun C, Sun Y, Song J, Li C, Li X, Zhang X, et al. Discovery of genes related to steroidal alkaloid biosynthesis in Fritillaria cirrhosa by generating and mining a dataset of expressed sequence tags (ESTs). Journal of Medicinal Plants Research. 2011; 5(21):5307-5314.

7. Bach TJ. Hydroxymethylglutaryl-CoA reductase, a key enzyme in phytosterol synthesis? Lipids. 1986; 21(1):82-88.

8. Suzuki M, Muranaka T. Molecular Genetics of Plant Sterol Backbone Synthesis. Lipids. 2007; 42(1):47-54.

9. Gondet L, Weber T, Maillot VP, Benveniste P, Bach TJ. Regulatory role of microsomal 3-hydroxy-3-methylglutaryl-coenzyme a reductase in a tobacco mutant that overproduces sterols. Biochem Biophys Res Commun. 1992; 186(2):888-893.

10. Sambrook J, Fritsch EF, Maniatis T. Molecular Cloning: A Laboratory Manual. Cold Spring Harbor laboratory press: CSH; 1982.

11. Tamura K, Dudley J, Nei M, Kumar S. MEGA4: molecular evolutionary genetics analysis (MEGA) software version 4.0. Molecular Biology and Evolution. 2007; 24(8):1596-1599.

12. Li P, Chen L. Xuezhikang inhibits the activity of HMG-CoA reductase in pig liver. Basic Medical Sciences and Clinics. 2003; 23:531-534.

13. Smith PK, Krohn RI, Hermanson GT, Mallia AK, Gartner FH, Provenzano MD, et al. Measurement of protein using bicinchoninic acid. Anal Biochem. 1985; 150(1):76-85.

14. Liu YJ, Zhang XN, Cheng QQ, Huang LQ, Gao W. Advances in studies on 3hydroxy-3-metllylglutaryl coenzyme A reductase in terpenoids biosynthesis of medicinal plants. Zhongguo Zhong Yao Za Zhi. 2013; 38(19):3226-3233.

15. Eti S, Saurabh P, Gaur AK. In silico characterization and differential expression pattern analysis of conserved HMG CoA reductase domain isolated from Aconitum balfourii Stapf. Biotech. 2016; 6(1):89-96.

16. Liao ZH, Tan QM, Chai YR, Zuo KJ, Chen M, Gong YF, et al. Cloning and characterisation of the gene encoding HMG-CoA reductase from Taxus media and its functional identification in yeast. Funct Plant Biol. 2004; 31(1):785-801.

17. Li HJ, Jiang Y, Li P. Characterizing distribution of steroidal alkaloids in Fritillaria spp. and related compound formulas by liquid chromatography-mass spectrometry combined with hierarchial cluster analysis. J Chromatogr A. 2009; 1216(3):2142-2149.

18. Hao DC, Gu XJ, Xiao PG, Peng Y. Phytochemical and biological research of Fritillaria medicine resources. Chinese Journal of Natural Medicines. 2013; 11(4):330-344.

19. Cong L, Ran FA, Cox D, Lin S, Barretto R, Habib N, et al. Multiplex genome engineering using CRISPR/Cas systems. Science. 2013; 339(6121):819-823. 
